\title{
LA COLLABORAZIONE TRA LABORATORIO DI MICROBIOLOGIA E TERAPIA INTENSIVA NELLO STUDIO ED ERADICAZIONE DI UN CLUSTER DI ACINETOBACTER BAUMANNII
}

*Leonetti P., *Costanzo S., *Falleni M., *Bartolino T., *Bagnoli M., *Casarosa S., *Pisaturo F., *Evangelista I., **Malacarne P.

\section{*Laboratorio Analisi I A.O.U.Pisa}

*** T. Intensiva P. Soccorso A.O.U. PISA

\section{Introduzione}

Presso il reparto di T. Intensiva A.O.U. Pisa si è verificato nel periodo novembre 2005- febbraio 2006 un cluster di Acinetobacter baumannii.

Il germe, inizialmente isolato nel liquido di drenaggio addominale di un paziente plurioperato, ha successivamente colonizzato a "macchia di leopardo" oltre il 70\% dei degenti in T. Intensiva.

Valutate senza risultati molte ipotesi relative alla modalità di trasmissione, si è ipotizzando che la contaminazione fosse imputabile ai ventilatori polmonari, e abbiamo ritenuto di effettuare controlli di sterilità.

Metodi

Sono state allestite colture di filtri antibatterici utilizzati nei ventilatori, deponendone una porzione in brodo cuore cervello e allestendo successive subculture su terreni agarizzati in piastre di Petri.

Due ventilatori sono risultati contaminati per Acinetobacter baumanni e una successiva campionatura più specifica di parti interne, ha permesso di rilevare una contaminazione della cella d'ossigeno.

\section{Risultati}

Le colture effettuate su sessanta filtri, hanno evidenziato contaminazioni batteriche ad opera di Staphilococcus ssp. e Pseudomonas ssp. in prevalenza.

Tra i germi isolati era presente anche nei filtri di 2 ventilatori polmonari un ceppo di Acinetobacter baumanni.. A seguito della sostituzione della cella d'ossigeno dei ventilatori e di un più oculato utilizzo dei filtri, il cluster è scomparso dopo circa 15 giorni.

Conclusioni

Questa esperienza di collaborazione ha permesso al clinico di seguire l'andamento delle colonizzazioni, di comprenderne la patogenesi e di verificarne la risoluzione, contenendo al minimo il danno clinico per i pazienti.

Il contributo del Laboratorio di Microbiologia è stato essenziale per la risoluzione del problema.

I risultati ottenuti sono il frutto di una sinergia elevata tra diverse professionalità, ove il ruolo del Laboratorio di Microbiologia assume una rilevanza assoluta. 